\title{
Effects of Torsion on Electromagnetic Fields
}

\author{
Laércio Dias \\ Laboratório de Física Teórica e Computacional, Departamento de Física, \\ Universidade Federal de Pernambuco, 50670-901, Recife, PE, Brazil \\ and Fernando Moraes \\ Laboratório de Física Teórica e Computacional, Departamento de Física, \\ Universidade Federal de Pernambuco, 50670-901, Recife, PE, Brazil and \\ Departamento de Física, CCEN, Universidade Federal da Paraíba, \\ Cidade Universitária, 58051-970 João Pessoa, PB, Brazil
}

Received on 14 February, 2005

\begin{abstract}
In this work, we investigate the effects of torsion on electromagnetic fields. As a model spacetime, endowed with both curvature and torsion, we choose a generalization of the cosmic string, the cosmic dislocation. Maxwell's equations in the spacetime of a cosmic dislocation are then solved, considering both the case of a static, uniform, charge distribution along the string, and the case of a constant current flowing through the string. We find that the torsion associated to the defect affects only the magnetic field whereas curvature affects both electric and magnetic fields. Moreover, the magnetic field is found to spiral up around the defect axis.
\end{abstract}

\section{INTRODUCTION}

The study of electromagnetism in a curved background has very important astrophysical implications as for example helping the understanding of the signals received from neutron stars and maybe also from black holes. Electromagnetic processes near such objects certainly will have generalrelativistic effects. After the generalization of Einstein's gravitational theory to include torsion, done by Hehl and coworkers [1], one might ask what are the effects of torsion on electromagnetic fields. In this work we study a simple but illustrative case: the electromagnetic field produced by a cyllindrically symmetric source coincident with a topological line defect that carries both curvature and torsion.

Topological structures like domain walls, strings and monopoles may have been produced by phase transitions involving spontaneous symmetry breaking in the early universe [2]. Such defects are associated to curvature singularities [3] and are solutions to Einstein's field equations. Although astronomical observations keep indicating that the macroscopic geometry of the universe is Riemannian it is possible that torsion may appear near curvature singularities [4]. Line defects containing torsion, like dislocations, appear within Einstein-Cartan-Sciama-Kibble gravitation theory [1] in Riemann-Cartan spacetime $U_{4}$.

We consider the cosmic dislocation [5] spacetime whose metric is given by

$$
d s^{2}=-d t^{2}+d r^{2}+\alpha^{2} r^{2} d \phi^{2}+(d z+\beta d \phi)^{2},
$$

in cylindrical coordinates. The parameter $\alpha$ is associated with the angular deficit of a cosmic string spacetime. The values of $\alpha$ are restricted to the interval $0<\alpha<1$, since the linear density of mass of a cosmic string, given by $\mu=(1-\alpha) / 4 G$, must be positive. The parameter $\beta$ is related to the torsion associated to the defect. For dislocations in solid state physics $\beta$ is related to the Burgers vector $\vec{b}$ by $\beta=\frac{b}{2 \pi}$.

This topological defect carries both torsion and curvature, both appearing as conical singularities on the $z$-axis. The only nonzero component of the torsion tensor in this case is given by the two-form [6]

$$
T^{z}=2 \pi \beta \delta^{2}(r) d r \wedge d \phi
$$

where $\delta^{2}(r)$ is the two-dimensional delta function. Analogously, the nonvanishing components of the curvature twoform are [6]

$$
R_{\phi}^{r}=-R_{r}^{\phi}=2 \pi \frac{(1-\alpha)}{\alpha} \delta^{2}(r) d r \wedge d \phi .
$$

This study intends to show how the changes introduced in the geometrical structure of spacetime by a topological line defect affect the solutions of Maxwell's equations. We are interested in the following two cases involving a cosmic dislocation: (i) the defect carries a density of charge and (ii) it carries a current. A similar problem was handled by M.F.A. da Silva et al. [7, 8], who calculated the magnetostatic field due to an electric current placed in the gravitational background of a rotating cosmic string. In their work, torsion comes from rotation, thus coupling time to the angular coordinate $\phi$. Here, torsion comes from the coupling between $\phi$ and $z$, as it is clear from Eq. (1). Other cases, with spherical symmetry, have appeared in the literature. For example,the electrostatic field and the potential of a point charge in the Schwartzschild metric obtained by Linet [9] and the magnetostatic field of a loop current around a black hole obtained by Petterson [10]. Related to these problems is the question of the self-force on electric and magnetic sources in the presence of topological defects, the object of much attention in recent years [11]-[19].

We restrict ourselves to the study of the generation of electric and magnetic fields by static sources. To facilitate the calculations, we consider the approximation [10] where the electromagnetic field is taken as a weak perturbation on the spacetime metric. Thus the influence of the metric on the electromagnetic field is much stronger than the influence of the electromagnetic field on the metric. In this approximation, the task of solving Einstein-Maxwell equations reduces to solving Maxwell equations in covariant form. 
This work is organized as follows. In Section II, we derive Maxwell equations in the cosmic dislocation spacetime. In Section III we solve them for the electrostatic field generated by a line of charge. We find that there is no effect of torsion on the electric field. On the other hand curvature amplifies it. In Section IV we calculate the magnetostatic field of a line current in the spacetime of the cosmic dislocation, finding an interesting effect: the peculiarities of the metric give rise to a $z$-component of the field. Finally, in Section V we we present our concluding remarks. We observe that in this paper we use geometrical units.

\section{MAXWELL EQUATIONS IN THE COSMIC DISLOCATION SPACETIME}

We start by writting Maxwell equations using differential forms:

$$
\mathbf{d F}=0
$$

and

$$
\star \mathbf{d} \star \mathbf{F}=\mathbf{J},
$$

where

$$
\mathbf{F}=\frac{1}{2} F_{\mu \nu} \mathbf{d} x^{\mu} \wedge \mathbf{d} x^{v}=\mathbf{B}+\mathbf{E} \wedge \mathbf{d} t
$$

is the Faraday two-form and $\mathbf{J}$ is the current density one-form given by

$$
\mathbf{J}=-\rho \mathbf{d} t+J_{r} \mathbf{d} r+J_{\phi} \mathbf{d} \phi+J_{z} \mathbf{d} z .
$$

In Eq. (6) the magnetic field is represented by the two-form $\mathbf{B}$ and the electric field by the one-form $\mathbf{E}$.

The Faraday two-form in terms of its components is therefore

$$
\begin{array}{r}
\mathbf{F}=F_{\phi z} \mathbf{d} \phi \wedge \mathbf{d} z+F_{z r} \mathbf{d} z \wedge \mathbf{d} r+F_{r \phi} \mathbf{d} r \wedge \mathbf{d} \phi \\
+F_{r t} \mathbf{d} r \wedge \mathbf{d} r+F_{\phi t} \mathbf{d} \phi \wedge \mathbf{d} t+F_{z t} \mathbf{d} z \wedge \mathbf{d} t .
\end{array}
$$

Eqs. (6) and (8) imply that

$$
\mathbf{B}=F_{\phi z} \mathbf{d} \phi \wedge \mathbf{d} z+F_{z r} \mathbf{d} z \wedge \mathbf{d} r+F_{r \phi} \mathbf{d} r \wedge \mathbf{d} \phi
$$

and

$$
\mathbf{E} \wedge \mathbf{d} t=F_{r t} \mathbf{d} r \wedge \mathbf{d} t+F_{\phi t} \mathbf{d} \phi \wedge \mathbf{d} t+F_{z t} \mathbf{d} z \wedge \mathbf{d} t,
$$

which leads to

$$
E_{r}=F_{r t} \quad E_{\phi}=F_{\phi t} \quad E_{z}=F_{z t}
$$

where

$$
\mathbf{E} \equiv E_{r} \mathbf{d} r+E_{\phi} \mathbf{d} \phi+E_{z} \mathbf{d} z
$$

We observe that the electric field vector components $\left(E^{r}, E^{\phi}, E^{z}\right)$ are related to the one-form $\mathbf{E}$ components $\left(E_{r}, E_{\phi}, E_{z}\right)$ by the metric in the usual way contravariant and covariant vector components are related. In the same way, the magnetic field vector components $\left(B^{r}, B^{\phi}, B^{z}\right)$ are related to a magnetic field one-form $\mathbf{B}_{1}$ components $\left(B_{r}, B_{\phi}, B_{z}\right)$. Nevertheless, the two-form $\mathbf{B}$ is related to $\mathbf{B}_{1}$ by the Hodge $\star$ operation :

$$
\star \mathbf{B}=\mathbf{B}_{1} \wedge \mathbf{d} t .
$$

Applying the Hodge $\star$ operator on Eq. (9) we obtain

$$
\begin{aligned}
\star \mathbf{B} & =\left(\frac{\alpha^{2} r^{2}+\beta^{2}}{\alpha r} F_{z r}+\frac{\beta}{\alpha r} F_{r \phi}\right) \mathbf{d} \phi \wedge \mathbf{d} t \\
& +\left(\frac{1}{\alpha r} F_{r \phi}+\frac{\beta}{\alpha r} F_{z r}\right) \mathbf{d} z \wedge \mathbf{d} t+\frac{F_{\phi z}}{\alpha r} \mathbf{d} r \wedge \mathbf{d} t .
\end{aligned}
$$

Therefore, we identify the components of the magnetic field one-form $\mathbf{B}_{1}$

$$
\begin{aligned}
B_{r} & =\frac{1}{\alpha r} F_{\phi z} \\
B_{\phi} & =\frac{\alpha^{2} r^{2}+\beta^{2}}{\alpha r} F_{z r}+\frac{\beta}{\alpha r} F_{r \phi} \\
B_{z} & =\frac{1}{\alpha r} F_{r \phi}+\frac{\beta}{\alpha r} F_{z r} .
\end{aligned}
$$

Now, applying the Hodge $\star$ operator on Eq. (10) we obtain

$$
\begin{aligned}
\star(\mathbf{E} \wedge \mathbf{d} t)= & -\alpha r F_{r t} \mathbf{d} \phi \wedge \mathbf{d} z \\
& -\left(\frac{1}{\alpha r} F_{\phi t}-\frac{\beta}{\alpha r} F_{z t}\right) \mathbf{d} z \wedge \mathbf{d} r \\
& -\left(\frac{\alpha^{2} r^{2}+\beta^{2}}{\alpha r} F_{z t}-\frac{\beta}{\alpha r} F_{\phi t}\right) \mathbf{d} r \wedge \mathbf{d} \phi .
\end{aligned}
$$

Finally, using Eqs. (6), (11), (14), (15) and (16), we obtain

$$
\begin{aligned}
& \star \mathbf{d} \star \mathbf{F}=\left\{\frac{1}{\alpha r} \frac{\partial B_{z}}{\partial \phi}-\frac{1}{\alpha r} \frac{\partial B_{\phi}}{\partial z}-\frac{\partial E_{r}}{\partial t}\right\} \mathbf{d} r \\
& +\left\{\frac{\beta}{\alpha r} \frac{\partial B_{\phi}}{\partial r}-\frac{\beta}{\alpha r} \frac{\partial B_{r}}{\partial \phi}+\frac{\alpha^{2} r^{2}+\beta^{2}}{\alpha r} \frac{\partial B_{r}}{\partial z}\right. \\
& \left.-\frac{\alpha^{2} r^{2}+\beta^{2}}{\alpha r} \frac{\partial B_{z}}{\partial r}-\frac{\partial E_{\phi}}{\partial t}\right\} \mathbf{d} \phi \\
& +\left\{\frac{1}{\alpha r} \frac{\partial B_{\phi}}{\partial r}-\frac{1}{\alpha r} \frac{\partial B_{r}}{\partial \phi}+\frac{\beta}{\alpha r} \frac{\partial B_{r}}{\partial z}-\frac{\beta}{\alpha r} \frac{\partial B_{z}}{\partial r}-\frac{\partial E_{z}}{\partial t}\right\} \mathbf{d} z \\
& -\left\{\frac{1}{r} \frac{\partial}{\partial r}\left(r E_{r}\right)+\frac{\partial}{\partial \phi}\left(\frac{1}{\alpha^{2} r^{2}} E_{\phi}-\frac{\beta}{\alpha^{2} r^{2}} E_{z}\right)\right. \\
& \left.+\frac{1}{\alpha^{2} r^{2}} \frac{\partial}{\partial z}\left[\left(\alpha^{2} r^{2}+\beta^{2}\right) E_{z}-\beta E_{\phi}\right]\right\} \mathbf{d} t .
\end{aligned}
$$

Care should be taken in interpreting the contravariant components of the fields since the metric (1) is associated to a nonorthonormal basis $\left(\vec{e}_{t}, \vec{e}_{r}, \vec{e}_{\phi}, \vec{e}_{z}\right)$, where $g_{\mu v}=\vec{e}_{\mu} \cdot \vec{e}_{v}$. Therefore we need to relate the components of the electric and magnetic field one-forms to the respective vectors in a normalized basis, such that in the no defect limit we recover the fields generated by a line source in flat spacetime. The new basis $\left(\vec{e}_{\hat{t}}, \vec{e}_{\hat{r}}, \vec{e}_{\hat{\phi}}, \vec{e}_{\hat{z}}\right)$ is simply obtained by

$$
\vec{e}_{\hat{\mu}}=\frac{\vec{e}_{\mu}}{\sqrt{g_{\mu \mu}}} .
$$


The components of a generic 1-form $\mathbf{A} \equiv A_{r} \mathbf{d} r+A_{\phi} \mathbf{d} \phi+$ $A_{z} \mathbf{d} z$ are related to the components of the equivalent vector $\vec{A}=A^{\hat{r}} \vec{e}_{\hat{r}}+A^{\hat{\phi}} \vec{e}_{\hat{\phi}}+A^{\hat{z}} \vec{e}_{\hat{z}}$, expressed in the normalized (but nonorthogornal) basis, by:

$$
\begin{gathered}
A_{r}=A^{\hat{r}} \\
A_{\phi}=\sqrt{\alpha^{2} r^{2}+\beta^{2}} A^{\hat{\phi}}+\beta A^{\hat{z}} \\
A_{z}=\frac{\beta}{\sqrt{\alpha^{2} r^{2}+\beta^{2}}} A^{\hat{\phi}}+A^{\hat{z}} .
\end{gathered}
$$

After some algebraic manipulations we finally obtain Eq. (5) in terms of the components of the electric, magnetic and current density vectors

$$
\begin{gathered}
\frac{1}{r} \frac{\partial}{\partial r}\left(r E^{\hat{r}}\right)+\frac{1}{\sqrt{\alpha^{2} r^{2}+\beta^{2}}} \frac{\partial E^{\hat{\phi}}}{\partial \phi}+\frac{\partial E^{\hat{z}}}{\partial z}=\rho \\
\frac{1}{\alpha r}\left(\frac{\beta}{\sqrt{\alpha^{2} r^{2}+\beta^{2}}} \frac{\partial}{\partial \phi}-\sqrt{\alpha^{2} r^{2}+\beta^{2}} \frac{\partial}{\partial z}\right) B^{\hat{\phi}} \\
+\frac{1}{\alpha r}\left(\frac{\partial}{\partial \phi}-\beta \frac{\partial}{\partial z}\right) B^{\hat{z}}=J^{\hat{r}}+\frac{\partial E^{\hat{r}}}{\partial t} \\
\frac{\sqrt{\left(\alpha^{2} r^{2}+\beta^{2}\right)}}{\alpha r}\left[\frac{\partial B^{\hat{r}}}{\partial z}-\frac{\partial}{\partial r}\left(B^{\hat{z}}+\frac{\beta}{\sqrt{\alpha^{2} r^{2}+\beta^{2}}} B^{\hat{\phi}}\right)\right] \\
\frac{1}{\alpha r}\left[\frac{\partial}{\partial r}\left(\sqrt{\alpha^{2} r^{2}+\beta^{2}} B^{\hat{\phi}}+\beta E^{\hat{\phi}}\right)-\frac{\partial B^{\hat{\phi}}}{\partial t}\right]=J^{\hat{z}}+\frac{\partial E^{\hat{z}}}{\partial t} .
\end{gathered}
$$

Notice that Eq. (22) corresponds to Gauss law and that Eqs. (23 - 25) correspond to Ampère-Maxwell law.

In a similar way, Eq. (4) leads to

$$
\begin{gathered}
\frac{1}{r} \frac{\partial}{\partial r}\left(r B^{\hat{r}}\right)+\frac{1}{\sqrt{\alpha^{2} r^{2}+\beta^{2}}} \frac{\partial B^{\hat{\phi}}}{\partial \phi}+\frac{\partial B^{\hat{z}}}{\partial z}=0 \\
\frac{1}{\alpha r}\left(\frac{\beta}{\sqrt{\alpha^{2} r^{2}+\beta^{2}}} \frac{\partial}{\partial \phi}-\sqrt{\alpha^{2} r^{2}+\beta^{2}} \frac{\partial}{\partial z}\right) E^{\hat{\phi}} \\
+\frac{1}{\alpha r}\left(\frac{\partial}{\partial \phi}-\beta \frac{\partial}{\partial z}\right) E^{\hat{z}}+\frac{\partial B^{\hat{r}}}{\partial t}=0 \\
\frac{\sqrt{\alpha^{2} r^{2}+\beta^{2}}}{\alpha r}\left[\frac{\partial E^{\hat{r}}}{\partial z}-\frac{\partial}{\partial r}\left(E^{\hat{z}}+\frac{\beta}{\sqrt{\alpha^{2} r^{2}+\beta^{2}}} E^{\hat{\phi}}\right)\right] \\
\frac{1}{\alpha r}\left[\frac{\partial B^{\hat{\phi}}}{\partial t}=0\right. \\
\left.\left[\sqrt{\alpha^{2} r^{2}+\beta^{2}} E^{\hat{\phi}}+\beta E^{\hat{z}}\right)-\frac{\partial E^{\hat{r}}}{\partial \phi}\right]+\frac{\partial B^{\hat{z}}}{\partial t}=0 .
\end{gathered}
$$

Now, Eq. (26) describes the absence of magnetic monopoles and Eqs. (27 - 29) correspond to Faraday law.

\section{ELECTRIC FIELD OF THE LINE CHARGE}

In this section we briefly discuss the case of a uniform line of charge coincident with the cosmic dislocation. In this case, the charge density is described by

$$
\rho(r)=\frac{\lambda}{2 \pi \alpha} \frac{\delta(r)}{r},
$$

where $\lambda$ is the linear charge density. The presence of $\alpha$ in this expression is due to the change in the volume element caused by the string metric.

The symmetries of the problem suggest that $E^{\hat{r}}=$ $E^{\hat{r}}(r), E^{\hat{\phi}}=E^{\hat{\phi}}(r)$ and $E^{\hat{z}}=E^{\hat{z}}(r)$. Eqs. (27-29) imply readily that

$$
E^{\hat{\phi}}(r)=E^{\hat{z}}(r)=0
$$

and Eq. (22) gives

$$
E^{\hat{r}}(r)=\frac{\lambda}{2 \pi \alpha} \frac{1}{r}
$$

This result might be explained by a simple argument based on the electric field lines, as follows. The process of creating the defect involves cutting out a wedge of space, which leaves less volume for the field lines to spread through. This increases the density of field lines therefore corresponding to an amplification of the electric field amplitude. This should be compared to the amplification found in the magnetostatic field of a current-carrying cosmic string [7]).

\section{MAGNETIC FIELD OF THE LINE CURRENT}

Now we treat the case where a current flows along the defect. The important equations now are $(23-25)$. Here the symmetry suggests that the nonvanishing components of the magnetic field are $B^{\hat{\phi}}=B^{\hat{\phi}}(r)$ and $B^{\hat{z}}=B^{\hat{z}}(r)$. With this, in the region $r>0$, Eqs. (24) and (25) turn into

$$
\begin{aligned}
& \frac{d}{d r}\left(B^{\hat{z}}+\frac{\beta}{\sqrt{\alpha^{2} r^{2}+\beta^{2}}} B^{\hat{\phi}}\right)=0 \\
& \frac{d}{d r}\left(\sqrt{\alpha^{2} r^{2}+\beta^{2}} B^{\hat{\phi}}+\beta B^{\hat{z}}\right)=0 .
\end{aligned}
$$

We have thus a coupled set of equations of very simple solution:

$$
B^{\hat{\phi}}(r)=k_{1} \frac{\sqrt{\alpha^{2} r^{2}+\beta^{2}}}{\alpha^{2} r^{2}}
$$

and

$$
B^{\hat{z}}(r)=k_{2}-\frac{\beta}{2 \pi \alpha^{2} r^{2}},
$$

where $k_{1}$ and $k_{2}$ are integration constants. In order to determine these constants we withdraw the defect by setting $\alpha=1$ 


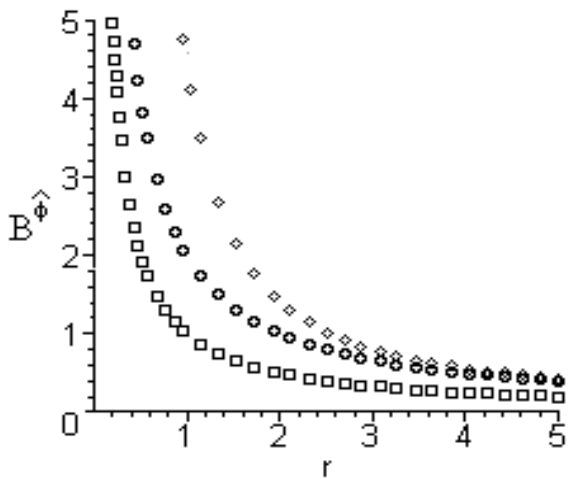

FIG. 1: $\phi$-component of the magnetic field: $\square$ in flat spacetime ( $\alpha=$ 1 and $\beta=0)$, $\circ$ in the cosmic string spacetime $(\alpha=0.5$ and $\beta=0)$, $\diamond$ in the cosmic dislocation spacetime $(\alpha=0.5$ and $\beta=1)$

and $\beta=0$. Thus, we recover the magnetic field of a line current in flat spacetime:

$$
\begin{gathered}
B_{\alpha=1, \beta=0}^{\hat{\phi}}(r)=\frac{I}{2 \pi r}, \\
B_{\alpha=1, \beta=0}^{\hat{z}}(r)=0,
\end{gathered}
$$

where $I$ is the electric current. Hence, we have $k_{1}=\frac{I}{2 \pi}$ and $k_{2}=0$. Substituting this into Eqs. (35) and (36), we finally get

$$
\begin{gathered}
B^{\hat{\phi}}(r)=\frac{I}{2 \pi} \frac{\sqrt{\alpha^{2} r^{2}+\beta^{2}}}{\alpha^{2} r^{2}}, \\
B^{\hat{z}}(r)=-\frac{I}{2 \pi} \frac{\beta}{\alpha^{2} r^{2}} .
\end{gathered}
$$

The coupling between the angular and the $z$ coordinates brings about an unexpected component of the magnetic field, which vanishes properly in the no torsion limit $\beta \rightarrow 0$. In Fig. 1 it is shown the $\phi$-component of the magnetic field in a few illustrative cases. In what follows we take a closer look at the magnetic field lines in this torsioned space.

In order to find the integral curves (magnetic field lines) of our vector (magnetic) field we need to solve the parametric system below:

$$
\begin{aligned}
\dot{r}(t) & =B^{r}(r, \phi, z) \\
\dot{\phi}(t) & =B^{\phi}(r, \phi, z) \\
\dot{z}(t) & =B^{z}(r, \phi, z),
\end{aligned}
$$

where $t$ is a parameter. Since $B^{r}=0, B^{\phi}=B^{\hat{\phi}} \sqrt{g_{\phi \phi}}$ and $B^{z}=$ $B^{\hat{z}}$ (see Eq. (18)) and with Eqs. (39) and (40) we have

$$
\begin{aligned}
\dot{r}(t) & =0 \\
\dot{\phi}(t) & =\frac{I}{2 \pi} \frac{1}{\alpha^{2} r^{2}} \\
\dot{z}(t) & =-\frac{I}{2 \pi} \frac{\beta}{\alpha^{2} r^{2}},
\end{aligned}
$$

whose solution is

$$
\begin{aligned}
r(t) & =r_{0} \\
\phi(t) & =\frac{I}{2 \pi \alpha^{2} r_{0}^{2}} t+\phi_{0} \\
z(t) & =-\frac{I \beta}{2 \pi \alpha^{2} r_{0}^{2}} t+z_{0},
\end{aligned}
$$

where $r_{0}, \phi_{0}, z_{0}$ are integration constants.

It is clear that the above set of equations describes a helix.

\section{CONCLUDING REMARKS}

In this work we investigated the influence of the torsion and curvature of a topological defect on electromagnetic fields generated by a line source coinciding with the defect. Torsion affects the magnetic field whereas curvature affects both electric and magnetic fields, but in different ways. Also, torsion forces the magnetic field lines to spiral up along the defect axis.

\section{Acknowledgments}

We are grateful to CNPq, FINEP(PRONEX) and CAPES (PROCAD) for partial support of this work.
[1] Friedrich W. Hehl et al., Rev. Mod. Phys. 48, 393 (1976).

[2] T. W. Kibble, J. Phys. A 9, 1387 (1976).

[3] D. D. Sokolov and A. A. Starobinskii, Sov. Phys. Dok1. 22, 312 (1977); Manuel Barriola and Alexander Vilenkin, Phys. Rev. Lett. 63, 341 (1989).

[4] William M. Baker, Class. Quantum Grav. 7, 717 (1990).

[5] D. V. Gal'tsov and P. S. Letelier, Phys. Rev. D 47, 4273 (1993).

[6] R. Puntigam and H. H. Soleng, Class. Quant. Grav. 14, 1129
(1997).

[7] A. G. R. Muriano, M. F. A. da Silva, Am. J. Phys. 65, 914 (1997).

[8] M. F. A. da Silva, O. T. da Silveira Filho, and J. A. Souza, Am. J. Phys. 57, 735 (1989).

[9] B. Linet, J. Phys. A 9, 1081 (1976).

[10] J. Petterson, Phys. Rev. D 10, 3166 (1974).

[11] B. Linet, Phys. Rev. D 33, 1833 (1986). 
[12] A. G. Smith, in Proceedings of Symposium on The Formation and Evolution of Cosmic String, edited by G. W. Gibbons, S. W. Hawking, and T. Vachaspati(Cambridge Unversity Press, Cambridge, England, 1990).

[13] E. R. Bezerra de Mello, V. B. Bezerra, C. Furtado, and F. Moraes, Phys. Rev. D 51, 7140 (1995).

[14] T. Souradeep and V. Sahni, Phys. Rev. D 46, 1616 (1992).

[15] M. E. X. Guimaraes and B. Linet, Class. Quant. Grav. 10, 1665 (1993).

[16] Claudio Furtado and Fernando Moraes, Class. Quantum Grav.
14, 3425 (1997).

[17] E. R. B. de Mello and C. Furtado, Phys. Rev. D 56, 1345 (1997).

[18] Sérgio Azevedo and Fernando Moraes, Phys. Lett. A 267, 208 (2000).

[19] A. M. de M. Carvalho, Claudio Furtado, and Fernando Moraes, Phys. Rev. D 62, 067504 (2000).

[20] A. Vilenkin, Phys. Rev. D 23, 852 (1981).

[21] C. W. Misner, K. S. Thorne, and J. A. Wheeler, Gravitation (Freeman, San Francisco, 1973). 